\title{
Manejo Protésico y Multidisciplinario de Prótesis Maxilofacial: Serie de Casos
}

\author{
Prosthodontic and Multidisciplinary Management \\ of Maxillofacial Prosthesis. A series of Cases
}

\author{
Mauricio Vivanco Barahona
}

VIVANCO, B. M. Manejo protésico y multidisciplinario de prótesis maxilofacial: Serie de casos. Int. J. Odontostomat., 15(4):797-805, 2021.

RESUMEN: Objetivo: determinar los principios de los tratamientos multidisciplinarios. Materiales y métodos: ejemplificar el trabajo multidisciplinario con 4 casos clínicos, tratamiento de pacientes complejos. Resultados: La evaluación multidisciplinar, delimita la dirección, planificación y tratamiento con opiniones discutidas y orientadas para el bien del paciente, definiendo los aspectos complementados para la rehabilitación. Discusión: El manejo multidisciplinario de los pacientes de Rehabilitación Maxilofacial presenta una compleja evaluación con relación a la planificación, pronóstico y tratamiento, donde diversos especialistas confluyen en una sinergia de criterios clínicos para un mejor resultado. Los recursos limitados en el servicio público incentivan el lograr los mejores resultados tanto con la disponibilidad económica del paciente como del servicio, esta limitación implica igualmente una gran oportunidad de trabajo conjunto. Se establecen finalmente, criterios generales y principios de tratamientos integrales. Conclusión: Para el trabajo en equipo multidisciplinario es fundamental mantener el objetivo de analizar y manejar en conjunto las distintas alternativas involucradas en los tratamientos de los casos, sean estas consideraciones Médicas, Odontológicas, Fonoaudiológicas, Psicólogas, Kinesiológicas, entre otras, para lograr el resultado funcional y estético deseado, satisfaciendo y tratando de recuperar a los pacientes de manera integral.

PALABRAS CLAVE: multidisciplinario, prótesis maxilofacial, manejo protésico, rehabilitación oral, cáncer de cabeza y cuello.

\section{INTRODUCCIÓN}

De las diferentes patologías que podemos encontrar en el territorio cráneo facial, existe una gran prevalencia del cáncer de cabeza y cuello, involucrando a su vez variados tipos de cáncer de la cavidad oral. El cáncer de cabeza y cuello ocupa el sexto lugar en todo el mundo, representando el 3,2 \% del total (Jemal et al., 2010; New DAHNO System, 2014). Otros estudios indican que el $5 \%$ de todas las patologías cancerígenas involucran a las estructuras de la boca, la lengua, la orofaringe, la nasofaringe y la laringe (Rieger et al. 2002). Sumado a lo anterior, hay tumores no cancerígenos y traumas que posterior a su extirpación o cicatrización, respectivamente, derivan en grandes secuelas craneofaciales, afectando en la calidad de vida del paciente, ya que cursan con angustia emocional y limita- ción de las funciones orales, aspectos que influyen en su relación hacia la sociedad. La remoción de las lesiones cancerígenas involucra alteraciones funcionales relacionadas con la fonación, masticación y deglución. La calidad de vida afectada presenta un componente psicológico involucrado debido a cambios en la apariencia y alteraciones psicosociales, dentro de los cambios postquirúrgicos (Newton et al., 1999).

La importancia del tratamiento rehabilitador, con una visión pre y post quirúrgica, requiere de un enfoque multidisciplinario y generalmente en un entorno institucional (Dudley et al., 2018). Este enfoque, en el mejor tratamiento, involucra a los cirujanos que deben realizan la remoción de tejidos y la reconstrucción coor- 
dinada con el Rehabilitador Maxilofacial (Huband, 2011). La Rehabilitación Oral es una de las 9 especialidades odontológicas reconocidas por la Asociación Dental Americana (ADA), siendo la Rehabilitación Maxilofacial una subespecialidad importante y reconocida (AAMP, 2020), componente clave de los programas de capacitación de posgrado (Dudley et al.). Los Protesistas Maxilofaciales se dedican al manejo correctivo de los defectos maxilofaciales adquiridos por la remoción quirúrgica de tumores, cicatrización de traumas, alteraciones congénitas o de crecimiento y desarrollo. A pesar de los casos, específicos por su grado de complejidad, el número de subespecialistas que realizan esta práctica clínica en forma activa es muy bajo (Huband). La prótesis maxilofacial debe restaurar y/o sustituir estructuras estomatognáticas y craneofaciales, intraorales y extraorales, maxilares, oculares y auriculares, con prótesis que pueden o no ser retiradas de manera regular o electiva (The Glossary of Prosthodontic Terms, 2017). Las prótesis maxilofaciales son realizadas por Rehabilitadores Maxilofaciales, y elaboradas por Técnicos Laboratoristas (Anaplastólogos), cuando la alternativa quirúrgica no pueda cumplir los objetivos reconstructivos y funcionales (Hatamleh et al., 2010), siendo la prótesis maxilofacial (somatoprótesis) la alternativa de tratamiento. El resultado depende de la interrelación del equipo y su tratamiento multidisciplinar médico - odontológico, limitando y faseando decisiones. El enfoque debe ser, por lo tanto, multidisciplinario y realizado generalmente en instituciones docentes u hospitalarias (Dudley et al.).

El objetivo de este trabajo fue ejemplificar 4 casos clínicos de tratamiento odontológico multidisciplinario de la Universidad de Valparaíso, en conjunto con la Armada de Chile y el Hospital Carlos Van Buren, tratamientos multidisciplinarios realizados en estos últimos servicios clínicos.

\section{REPORTE DE CASOS}

CASO 1: Paciente 71 años, sexo femenino, ingresa (2018) al Hospital Naval Almirante Nef, con lesión crónica de lengua, biopsia diagnóstica de Carcinoma Espino Celular de poca diferenciación, grado 3 , ubicado en dorso y zona ventral izquierda de lengua, sin compromiso óseo ni piso de boca. Se realizó resectomía parcial de lengua e injerto libre microvascularizado (Fig. 1) de piel antebrazo izquierdo (Fig. 2). Paciente desdentado parcial maxilar superior e inferior, con alto grado de dificultad en la fonación, trituración y deglución de alimentos.
Sin indicación de quimio ni radioterapia. Se indica somatoprótesis removible superior con descenso de paladar duro para lograr contacto funcional con lengua y reconstrucción de injerto en ésta (presenta anatomía estable). Para esto se realizaron las restauraciones necesarias y ajuste oclusal, confeccionando una prótesis parcial acrílica en forma inicial para evaluar funcionamiento, adaptación y estabilidad de tejidos. Posteriormente se agregó material de rebasado temporal (Lynal®,

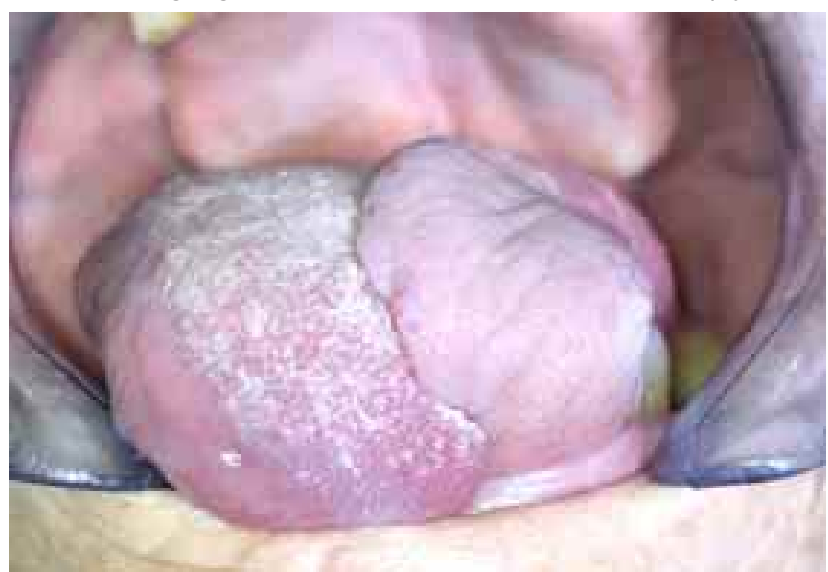

Fig. 1. Visión frontal de la lengua, donde se evidencia el injerto realizado posteriormente a resección y reconstrucción lingual izquierda.

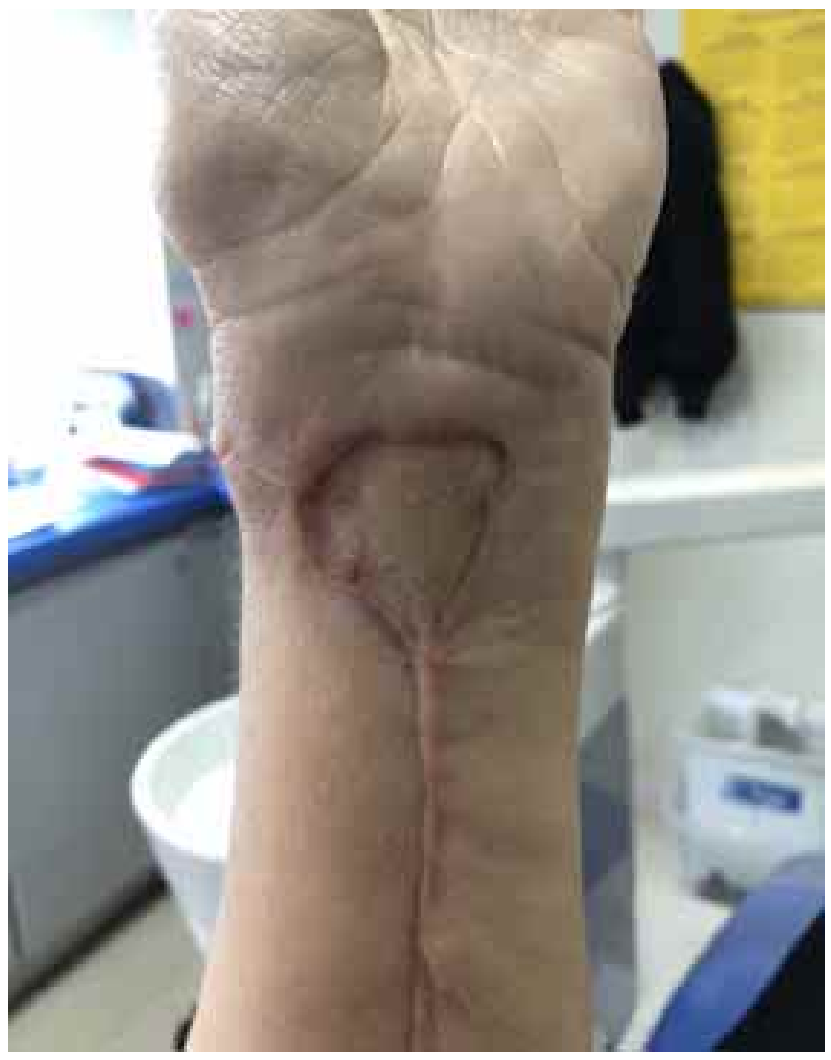

Fig. 2. Zona donante de piel antebrazo izquierdo de la paciente para injerto lingual. 
Dentsply Sirona®) (Fig. 3), para producir en el material temporal una impronta lingual funcional inicial, y adaptación al ser usada. La colocación del material temporal se ubicó solo en el paladar protésico maxilar superior zona izquierda. Posterior a eso, mejora eficiencia masticatoria en forma considerable, fonación y deglución. Se procede a transformación de material temporal a resina acrílica. La paciente fue derivada a tratamiento fonoaudiológico durante el proceso, control médico y odontológico para planificación de rehabilitación maxilar inferior en base a implantes óseointegrados.

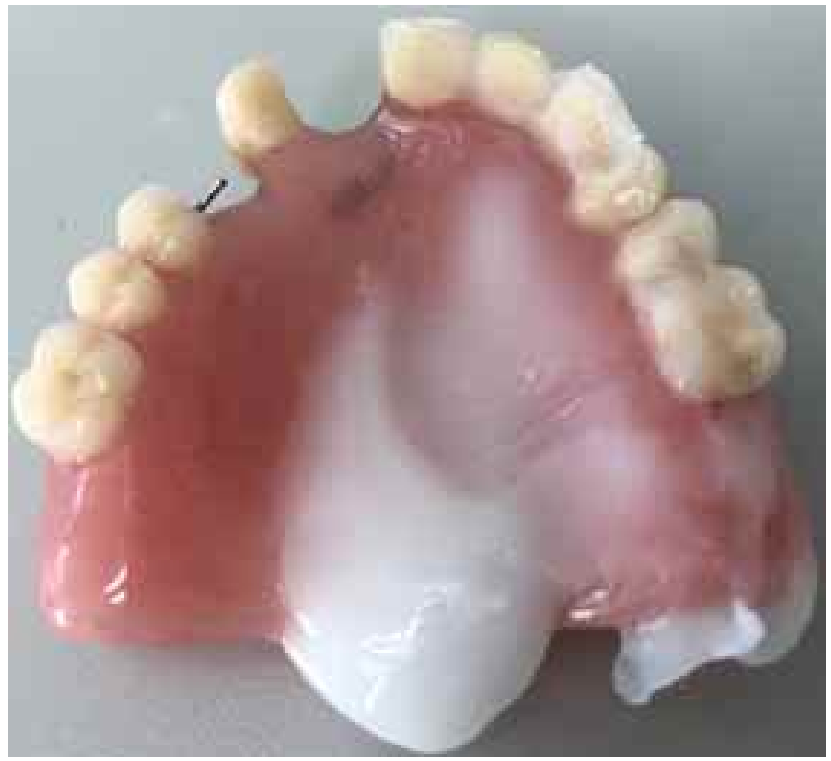

Fig. 3. Colocación de material de rebasado temporal (Lynal囚) para la impronta funcional lingual.

CASO 2: Paciente 55 años, sexo femenino, diagnóstico previo (1997) Tumor de Células Gigantes, maxilar superior, en zona ósea posterior izquierda, comprometiendo fosa nasal y piso de órbita. Se efectuó resección total de zonas descritas (Fig. 4). Paciente en control por 20 años, sin recidiva. Se realiza rehabilitación maxilar superior, recambio por inestabilidad de prótesis obturadora metal-acrílica antigua, sin retención, de gran peso y que no presenta sello protésico en comunicación maxilar izquierdo, posterior a D.2.3 con comunicación amplia a fosa nasal y sin comunicación por sello mucoso de perforación parcial de piso de órbita (Fig. 5). Se regulariza oclusión, exodoncias de dientes con escaso soporte periodontal y lesiones apicales. Se realiza prótesis fija plural de grupo II. Uso de prótesis obturadora provisoria acrílica transitoria por 6 meses (Fig. 6 y 7). Actualmente en evaluación para prótesis maxilofacial definitiva y reconstrucción ósea estructural de piso de órbita, considerando alternativa quirúrgica de cierre de paladar.

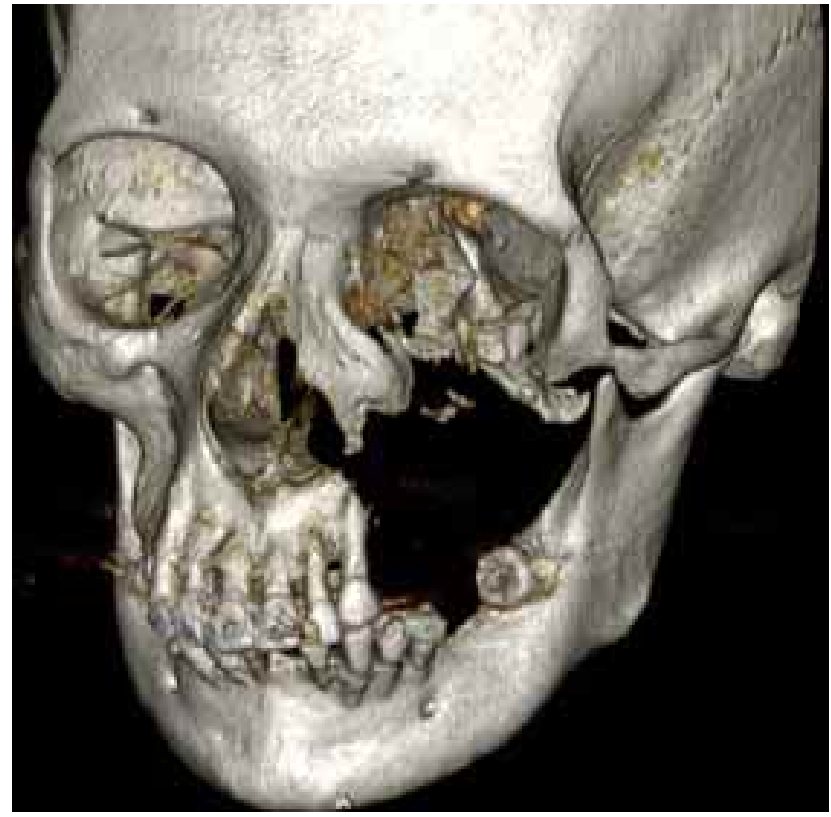

Fig. 4. Reconstrucción volumétrica (VR) cráneo facial, post quirúrgica.

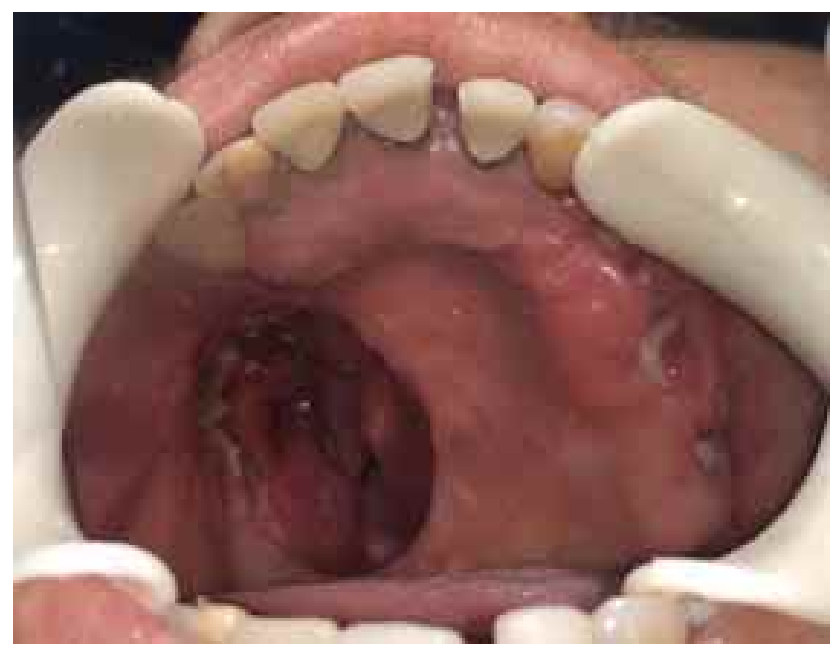

Fig. 5. Inicio de tratamiento, mala higiene oral, comunicación buco nasal por resección parcial maxilar.

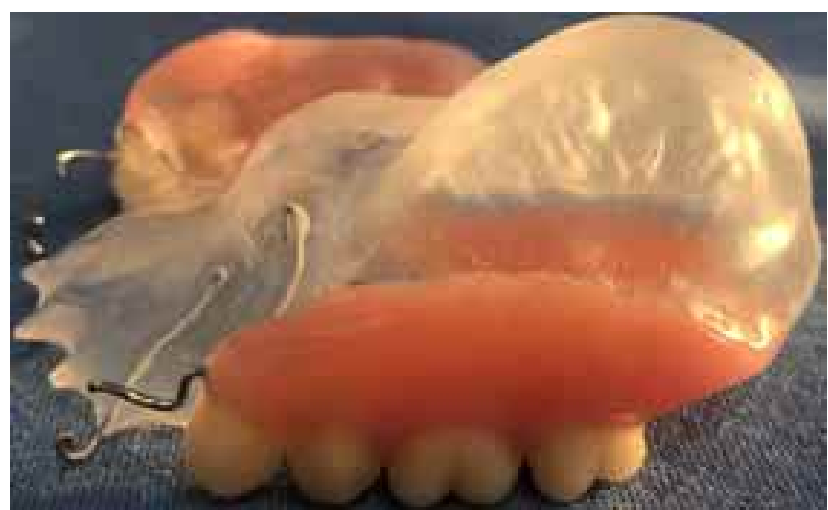

Fig. 6. Prótesis temporal, con protuberancia para obturación de comunicación buco nasal. 
CASO 3: Paciente 41 años, sexo femenino. Diagnóstico (2016): Tumor de Células Gigantes, extensa lesión osteolítica zona maxilar inferior izquierda, sin compromiso de ángulo mandibular. Cirugía de resección y colocación en la misma cirugía de injerto autólogo (peroné) y 03 placas de fijación maxilar (Fig. 8). 2 años de control. Se realiza planificación (Fig. 9 y 10) y posteriormente cirugía de instalación de 3 implantes óseointegrados (2018) en zona planificada en conjunto con la evaluación multidisciplinaria protésica, para rehabilitación zona D.3.5, 3.6 y 3.7.

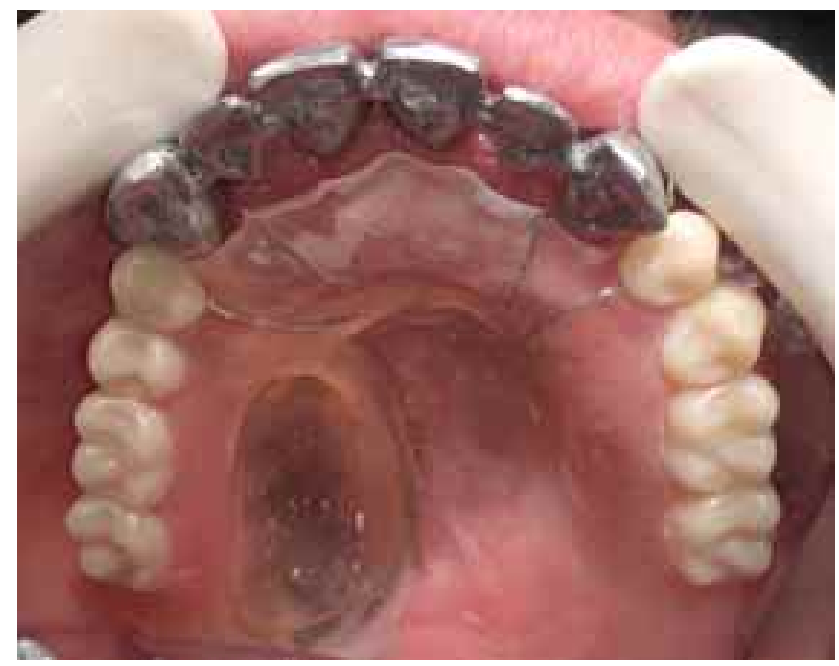

Fig. 7. Tratamiento en etapa intermedia, con prótesis obturadora en posición

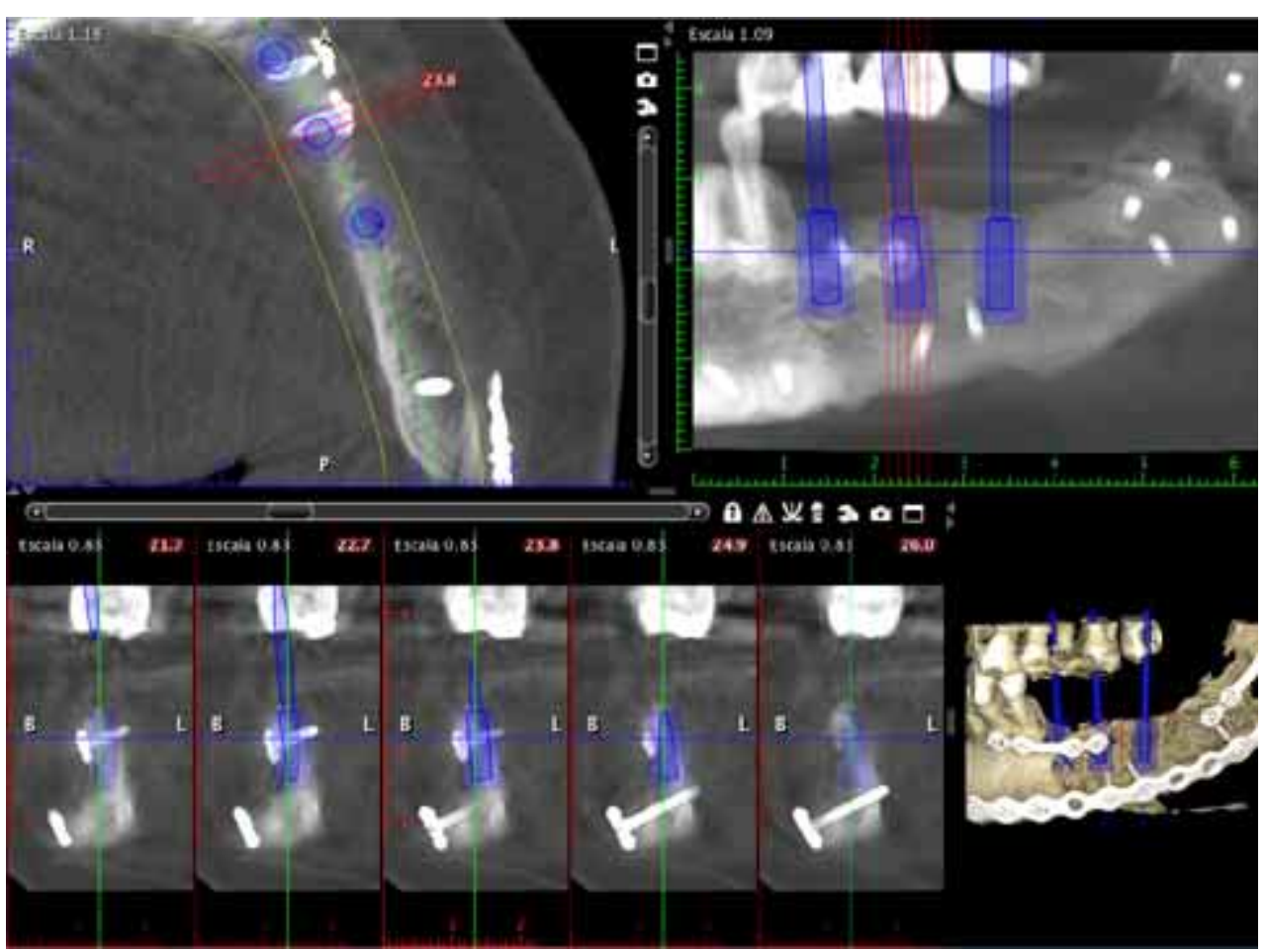

800
CASO 4: Paciente 67 años, sexo masculino, consulta por dolor zona maxilar superior izquierda, no remitente a tratamiento endodóntico (2018). Ante consulta posterior y exámenes complementarios se establece el diagnóstico de: Carcinoma Espino Celular, No Queratinizante de seno maxilar izquierdo. Comité oncológico multidisciplinario indica resección hemimaxilar completa. Cirugía involucra línea media y reborde anterior y $2 / 3$ de paladar duro, sin involucrar paladar blando. En la misma intervención quirúrgica se instala injerto libre microvascularizado de piel en zona paladar izquierdo perforado post resección, obturando completamente comunicación oro - nasal (Fig. 11). No se indica colocación de implantes dentales para evitar complicaciones quirúrgicas asociadas a quimio-

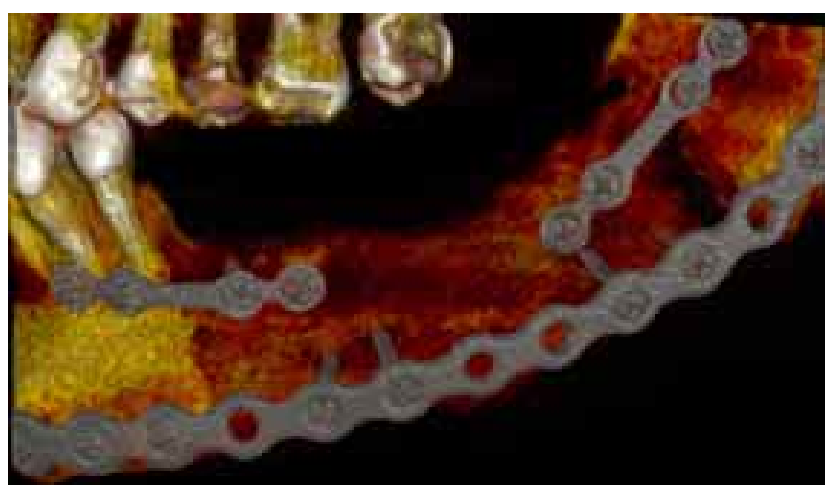

Fig. 8. Reconstrucción volumétrica de escáner zona mandibular izquierda, mostrando injerto óseo en bloque de peroné y placas de fijación quirúrgica.
Fig. 9. Planificación de implantes, evitando contacto con placas quirúrgicas y tornillos de fijación. 


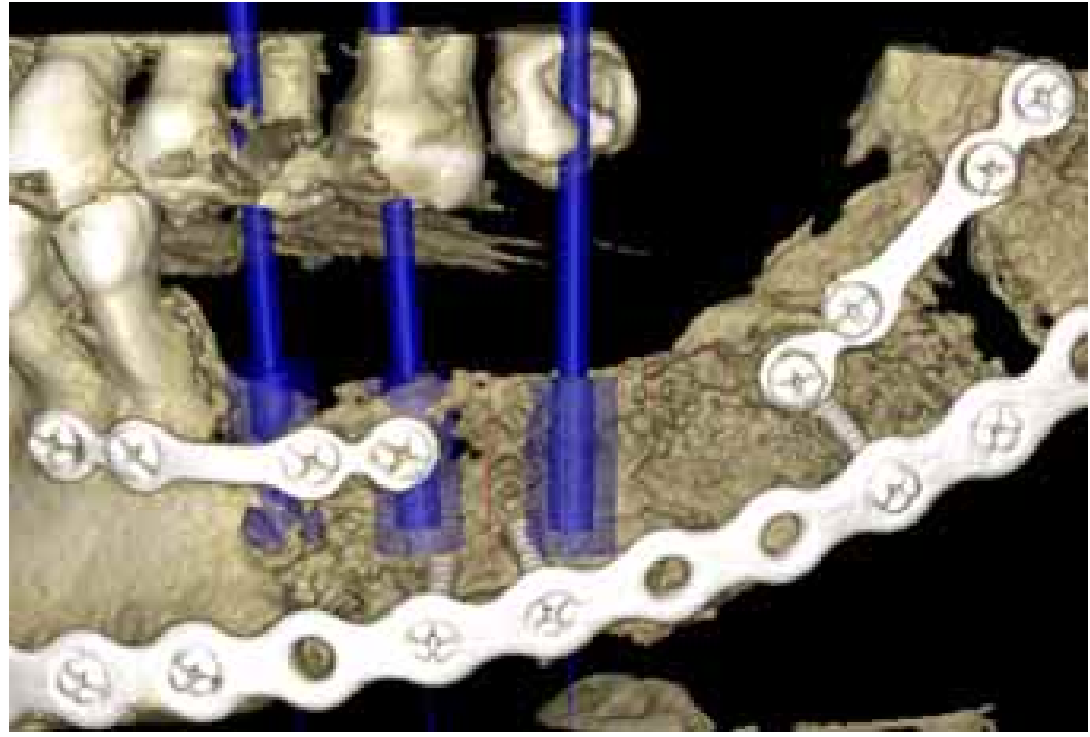

Fig. 10. Zona definida para colocación 03 implantes.

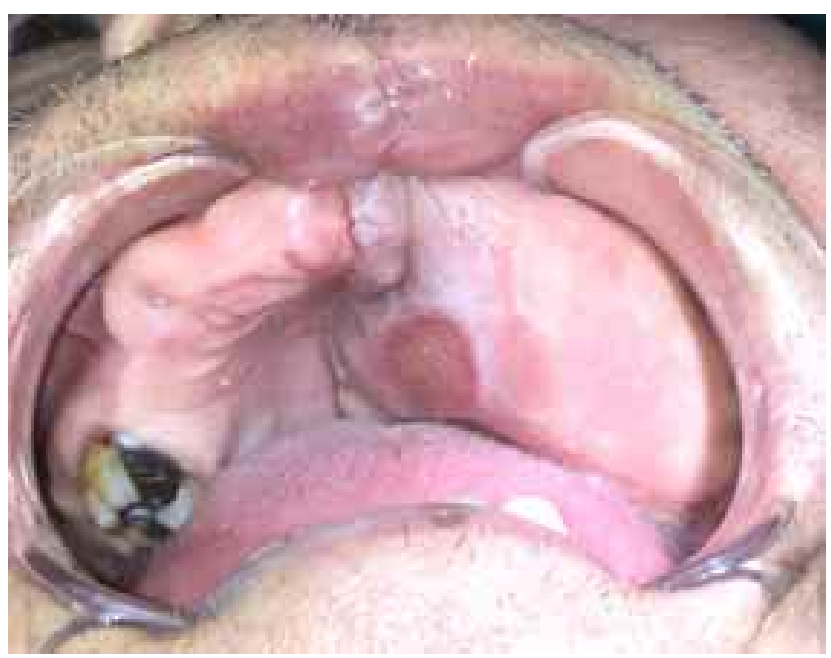

Fig. 11. Vista anteroinferior de injerto de piel en ubicación paladar izquierdo, sin soporte óseo.

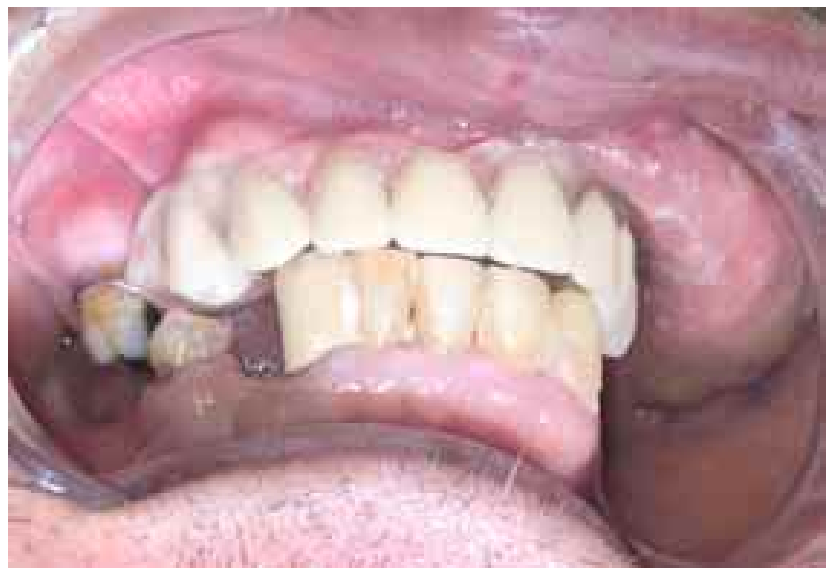

Fig. 12. Prótesis transitoria para soporte de injerto en cicatrización. terapia posterior. En los controles protésicos se instala somatoprótesis maxilar acrílica cosmética adhesiva, con retenedor en D.1.7, para soporte de injerto vía intraoral, el que en su proceso de cicatrización obstruye gran parte de la cavidad oral. Estabilidad protésica compleja, falta de retención, estética adecuada (Fig. 12). Se realizan controles sucesivos para evaluación multidisciplinar, y control de cicatrización intraoral y extraoral.

\section{RESULTADOS}

El manejo de rehabilitación maxilofacial en estos 4 casos clínicos involucra decisiones médicas - odontológicas en conjunto. El tipo de lesiones descritas, su agresividad, tipo de resección realizada, cantidad y calidad de tejido remanente, tratamientos médicos complementarios y la planificación protésica, con sus objetivos funcionales y estéticos delimitan la variabilidad de caminos a seguir. Los casos presentados ejemplifican el trabajo multidisciplinario, interinstitucional.

\section{DISCUSIÓN}

La interrelación multidisciplinaria se logra fundamentalmente cuando hay un objetivo en común: rehabilitar a un paciente en sus aspectos médicos odontológicos, con éxito funcional y estético. El manejo multidisciplinario de los pacientes de rehabilitación maxilofacial presenta desafíos en la planificación y pronóstico, donde diversos especialistas deben confluir en una sinergia de criterios clínicos y acuerdos. Además, se debe lograr un compromiso individual y familiar del paciente, debido a la larga duración, afección psicológica y económica de los tratamientos.

Los resultados son variables y dependientes de la complejidad de los tratamientos; la eficacia de éstos y la probabilidad de supervivencia de los pacientes, deben equilibrarse con el funcionamiento potencial protésico y la calidad de vida (Quality of Life, QoL) (Mehanna et al., 2010; Weller et al., 2010). Estudios acerca de la calidad de vida de los pacientes con cáncer de cabeza y cuello demostraron altos niveles de angustia emocional, asociados a complicaciones por li- 
mitaciones físicas, como alteraciones en la imagen personal y en sus relaciones sociales (Markt \& Lemon, 2001; Shoen et al., 2001). Se ha descrito que las técnicas de reconstrucción quirúrgica, rehabilitación protésica, o una combinación de ambas para corregir la desfiguración facial puede mejorar la función, la autoestima, la confianza y el bienestar psicológico del paciente (Roumanas et al., 2002; Guttal et al., 2006), pero éxito es dependiente de que diversas especialidades se involucren en el manejo óptimo es multidisciplinario. Se describen como pilares del tratamiento la cirugía, la radioterapia y la quimioterapia, incluyendo Odontólogos especialistas (NICE, 2004), todos enfocados en la rehabilitación final, ya que, sin la planificación adecuada, la mutilación de los pacientes y la desfavorable calidad de vida es inminente.

El enfoque multidisciplinario debe incluir una ablación quirúrgica y reconstrucción dispuesta a recibir el trabajo del Protesista Maxilofacial. La planificación prequirúrgica y posquirúrgica son tan importantes como los tratamientos complementarios (quimioterapia, radioterapia, tratamiento psicológico, fonoaudiológico, entre otros), todo debe ser coordinado por el especialista responsable antes del procedimiento quirúrgico: planificación y trabajo multidisciplinario, son la clave, la coordinación elimina la patología, minimiza las secuelas y el resultado posquirúrgico tiende a ser favorable (Lemon et al., 2005; Salinas, 2010).

La denominación "equipo" delimita la dirección de los especialistas hacia un objetivo único: el paciente y su familia. El actuar en conjunto permite a los especialistas aplicar individualmente sus conocimientos y visiones (Sánchez-Ruiz et al., 1999), generando espacios de crecimiento profesional, discutiendo con opiniones orientadas al bien integral del paciente y sin la visión sesgada de su propia especialidad, la supervisión constante, reevaluación y opinión del paciente deben ser consideradas igualmente.

Los recursos limitados en el servicio público estimulan el lograr los mejores resultados, ofreciendo una gran oportunidad de trabajo conjunto para la resolución de estos casos complejos. Las reuniones periódicas multidisciplinares requieren necesariamente un coordinador, el que debe tener un liderazgo flexible en las distintas fases; un equipo igualitario, con interacción de decisiones es mejor que uno autoritario. Además, un equipo oficialmente constituido desarrolla responsabilidades clínicas y legales compartidas e informadas (Sánchez-Ruiz et al.). Los posibles fracasos parciales deben ser estimados en la planificación, redireccionando para el logro de objetivos, estableciendo un proceso dinámico; aún así deben plantearse todas las alternativas desde la primera consulta. involucran:

Los principios de tratamientos integrales

1. Consensuar diagnóstico y planificación temprana, siguiendo las directrices multidisciplinarias establecidas.

2. Correlacionar costo/beneficio/resultado/pronóstico de cada alternativa de tratamiento.

3. Registrar autorización de tratamiento escrita y verbalmente por los pacientes y sus familias (consentimiento informado).

4. Reevaluar, redireccionar y consensuar el tratamiento.

5. Protocolizar el seguimiento en reuniones multidisciplinarias de evolución.

6. Establecer limitaciones discutidas para evitar y prevenir daños.

Puede haber implicancias faciales en los tratamientos de los tumores región de la cabeza y cuello, requiriendo necesariamente cirugía que derivará en una anatomía alterada con trastornos en la función oral y la calidad de vida. Si además se consideran intervenciones como radioterapia, las condiciones de los tejidos empeorarán aún más, ya que se producirá una retracción de los tejidos faciales remanentes y otras secuelas orales inducidas por la radiación, lo que complica el pronóstico de la rehabilitación. La coordinación de todas las especialidades incluye al equipo de oncología (Balasubramanian et al., 2007). Siempre el objetivo es la vida del paciente y su recuperación.

Las prótesis maxilofaciales tienen como objetivo principal reconstructivo recuperar la forma y función normal. En los pacientes mutilados, la reconstrucción maxilar, mandibular, ocular, facial o auricular involucra forma y función, no solo la estética facial, por lo que hay que considerar otros factores como la rehabilitación dental (Salman et al., 2017).

Al requerir una maxilectomía completa o parcial maxilar o mandibular, el enfoque debe ser con mayor hincapié multidisciplinario. El tratamiento protésico de los pacientes con defectos en el paladar duro o blando es complejo, especialmente en maxilares desdentados, debido a la imposibilidad de obtener retención o un adecuado sello en el borde, además de la deficiente superficie de apoyo palatina y el aumento de peso protésico (Tuna et al., 2009). Grandes o pe- 
queños defectos en el paladar resultan en alteraciones de la fonación, masticación, olfato y gusto (Rogers et al., 2003; Ortegon et al., 2008), además la comunicación del paladar hacia las fosas nasales afecta en forma inicial en la alimentación, succión, deglución, y produce posteriormente una resonancia nasal excesiva al hablar (voz hipernasal) (Dudley et al.).

Una prótesis maxilofacial obturadora palatina mejora inmediatamente la fonación ya que permite solamente la resonancia de la cavidad oral, separándola de cavidad nasal, minimizando la hipernasalidad (Haug, 2007), disminuye el traspaso de fluido oral a la cavidad nasal, mejora la deglución y complementariamente la masticación y estética al reemplazar los dientes extraídos durante la resección quirúrgica (lo que no siempre es prioritario).

Hay casos en que la planificación protésica debe considerar algunas remodelaciones anatómicas para utilizar concavidades, colocación de injertos óseos o implantes dentales. Estas consideraciones deben ser establecidas en un examen prequirúrgico exhaustivo y una consulta multidisciplinaria para un tratamiento combinado quirúrgico-protésico muy definido, pensando en una óptima adaptación funcional protésica y mejor calidad de vida. Debemos considerar que la cirugía de extirpación de cáncer genera malformaciones, al igual que los traumas, dando lugar a defectos faciales extensos que no pueden ser cubiertos por injertos, produciendo múltiples problemas funcionales y alteraciones psicológicas independiente de la edad del paciente (Goiato et al., 2009). Los obturadores y las prótesis faciales son importantes para la resocialización del paciente, el nivel de reintegración está directamente relacionado al grado de satisfacción del uso de la rehabilitación, por lo tanto, el Protesista Maxilofacial debe esforzarse en la satisfacción del paciente durante el tratamiento (Goiato et al.).

Los métodos de retención más utilizados para las prótesis maxilofaciales son los adhesivos. En la actualidad, los implantes (intraorales o faciales) suelen implementarse como un procedimiento secundario en las prótesis maxilofaciales. El aumento de su uso, asociado a barras o imanes retenidos por implantes, permiten una retención de el doble de tiempo que las prótesis retenidas solamente por implantes (Hatamleh et al.). Diferentes tecnologías digitales son utilizadas por un tercio de los Protesistas en la fabricación de las rehabilitaciones maxilofaciales (Hatamleh et al.). La planificación quirúrgica virtual, para placas de reconstrucción individualizadas, guías quirúrgicas de implantes para optimizar resultados en una sola intervención (Shoen et al.) y otras técnicas complementarias como los colgajos libres microvascularizados pueden utilizarse para optimizar las maniobras reconstructivas, detalles de una planificación quirúrgica compleja (Cunningham \& Gal, 2008). Lo más importante de la colaboración quirúrgica en beneficio de la rehabilitación es la comunicación en el período preoperatorio y en el intraoperatorio (Cunningham \& Gal), en la implementación de las técnicas quirúrgicas y en la clasificación de los defectos quirúrgicos maxilares (Haug).

\section{CONCLUSIÓN}

El trabajo en equipo multidisciplinario tiene diversas ventajas. Es fundamental en la rehabilitación maxilofacial el análisis de casos y manejo en conjunto médico - odontológico. Estos casos son siempre de alta complejidad, ya que requieren coordinación y acuerdos de tratamiento en especialidades tan diversas como Oncología, Radioterapia, Cirugía de Cabeza y Cuello, Cirugía Maxilofacial, Rehabilitación Maxilofacial entre otras, además del complemento con tratamiento Fonoaudiológico y Psicológico, para lograr el resultado funcional y estético deseado, satisfaciendo al paciente y tratando de recuperarlo integral y socialmente. Este trabajo multidisciplinario debe buscar la colaboración de varios profesionales con diferentes tipos de especialización (Haug).

Analizadas las diferentes situaciones clínicas, la rehabilitación maxilofacial se presenta como una alternativa de buen pronóstico, sobre todo en los casos que en una reconstrucción quirúrgica - funcional - estética facial no pueda realizarse debido a las condiciones psicofísicas del paciente o debido a una pérdida excesiva de tejidos, derivada por una planificación quirúrgica con pobres resultados (Leonardi et al., 2008). La prótesis maxilofacial presenta varias ventajas sobre una intervención quirúrgica reconstructiva posterior, ya que implica la restauración de elementos del habla normal, masticación, funciones de deglución y la estética (Beumer III et al., 2011; Kim et al., 2016), como por ejemplo en el caso de los obturadores al crear un sello artificial entre las cavidades oral y nasal, facilitando los cambios en la presión del aire oral (Abreu et al., 2007).

En los casos mostrados y en prótesis faciales, no importan solamente la función y la estética, sino también el efecto que cumplen en la sociabilización del 
paciente, dado por el grado de satisfacción de la rehabilitación realizada. Debemos estar atentos a los requerimientos de los pacientes, escucharlos a ellos y a sus familias, durante y después del tratamiento. Los estudios relacionados al grado de satisfacción de prótesis maxilofaciales (Goiato et al.), indican que los problemas experimentados por estos pacientes disminuyen cuando los especialistas los mantienen en un control constante. La rehabilitación maxilofacial es efectiva en cuanto a la funcionalidad, estética y bienestar al reinsertar a los pacientes en su entorno social y familiar (Goiato et al.).

Los casos presentados ilustran las complejidades que involucra realizar prótesis maxilofacial, difiriendo ampliamente en técnicas, planificación, cuidado de los aspectos psicológicos y multidisciplinarios de cada rehabilitación. Finalmente, para el clínico la satisfacción en la resolución de los casos se encontrará en la respuesta de los pacientes frente a los resultados obtenidos (Dudley et al.)

En resumen, la colaboración y trabajo en equipo mejora los objetivos planteados en la reconstrucción de cabeza y cuello, tanto para el paciente, como para los equipos quirúrgicos - protésicos y multidisciplinarios.

\section{AGRADECIMIENTOS}

El autor desea expresar su reconocimiento a los Doctores Jacqueline Maritano V. y Nelson Dib G. del Servicio de Cirugía Oral y Maxilofacial del Hospital Naval Almirante Nef y al Dr. Víctor Moraga G. del Hospital Carlos Van Buren, por colaboración en el desarrollo de los casos presentados.

VIVANCO, B. M. Prosthodontic and multidisciplinary management of maxillofacial prosthesis: A series of cases. Int. J.Odontostomat., 15(4):797-805, 2021.

ABSTRACT: The objective of this study was to determine the principles of multidisciplinary treatments, and to exemplify multidisciplinary work with 4 clinical cases, treatment of complex patients. The multidisciplinary evaluation, delimits the direction, planning and treatment with discussed and oriented opinions for the benefit of the patient, defining the complemented aspects for the rehabilitation. The multidisciplinary management of patients in Maxillofacial Rehabilitation presents a complex evaluation in relation to planning, prognosis and treatment, where different specialists converge in a synergy of clinical criteria, for a better outcome.
The limited resources in the public service encourage the achievement of the best results both with the economic availability of the patient and the service; this limitation also implies a great opportunity to work together. Finally, general criteria and principles of integral treatments are established. For the multidisciplinary team work it is fundamental to maintain the objective of analyzing and managing together the different alternatives involved in the treatment of the cases, medical and dental considerations, phonoaudiology, psychology, kinesiology, among others, to achieve the desired functional and aesthetic result, satisfying and trying to recover the patients integrally.

KEY WORDS: multidisciplinary, maxillofacial prosthesis, prosthodontic management, oral rehabilitation, head and neck cancer.

\section{REFERENCIAS BIBLIOGRÁFICAS}

Abreu, A.; Levy, D.; Rodriguez, E. \& Rivera, I. Oral rehabilitation of a patient with complete unilateral cleft lip and palate using an implantretained speech-aid prosthesis: clinical report. Cleft Palate Craniofac. J. 44(6):673-7, 2007.

American Academy of Maxillofacial Prosthetics (AAMP). 2020. Disponible en: https://www.maxillofacialprosthetics.org/what-is-mp.html

Balasubramanian, S; van Oort, R. P.; de Bont, L. G. M. Maxillofacial rehabilitation in head and neck oncology. Int. J. Oral Maxillofac. Surg., 36(11):014.37, 2007.

Beumer III, J.; Marunick, M. T.; Esposito S. J. Maxillofacial rehabilitation: prosthodontic and surgical management of cancer related, acquired, and congenital defects of the head and neck. 3rd ed., Chicago, Quintessence, 2011.

Leonardi, A.; Buonaccorsi, S.; Pellacchia, V.; Moricca, L. M.; Indrizzi, E. \& Fini, G. Maxillofacial prosthetic rehabilitation using extraoral implants. J. Craniofac. Surg., 19(2):398-405, 2008.

Cunningham, Jr, L. \& Gal, T. J. S111: Interdisciplinary reconstruction of complex facial defects. J. Oral Maxillofac. Surg., 66(8 supp):144, 2008.

Dudley, J.; Mughal, F.; Hotinski, E.; Mahmud, M. Prosthodontic management of maxillofacial cases: a case series. Aust. Dent. J., 63(1):124-8, 2018.

Goiato, M. C.; Pesqueira, A. A.; Ramos da Silva, C.; Gennari Filho, H. \& Micheline Dos Santos, D. Patient satisfaction with maxillofacial prosthesis. Literature review. J. Plast. Reconstr. Aesthet. Surg., 62(2):175-80, 2009.

Guttal, S. S.; Patil, N. P. \& Shetye, A. D. Prosthetic rehabilitation of a midfacial defect resulting from lethal midline granuloma-a clinical report. J. Oral Rehabil., 33(11): 863-7. 2006.

Hatamleh, M. M.; Haylock, C.; Watson, J. \& Watts, D. C. Maxillofacial prosthetic rehabilitation in the UK: a survey of maxillofacial prosthetists' and technologists' attitudes and opinions. Int. J. Oral Maxillofac. Surg., 39(12):1186-92. 2010.

Haug, S. P. Maxillofacial prosthetic management of the maxillary resection patient. Atlas Oral Maxillofac. Surg. Clin. North Am., 15(1):51-68, 2007

Huband, M. Prosthetic rehabilitation. Dermatol. Clin., 29(2):325-x, 2011.

Jemal, A.; Siegel, R.; Xu, J. \& Ward, E. Cancer statistics, 2010. CA Cancer J. Clin., 60(5):277-300, 2010.

Kim, J. H.; Shin, S. Y.; Paek, J.; Lee, J. H. \& Kwon, H. B. Analysis of maxillofacial prosthetics at university dental hospitals in the capital region of Korea. J. Adv. Prosthodont., 8(3):229-34, 2016. 
Lemon, J. C.; Kiat-amnuay, S.; Gettleman, L.; Martin, J. W. \& Chambers, M. S. Facial prosthetic rehabilitation: preprosthetic surgical techniques and biomaterials. Curr. Opin. Otolaryngol, Head Neck Surg., 13(4):255-62, 2005.

Markt, J. C. \& Lemon, J. C. Extraoral maxillofacial prosthetic rehabilitation at the M. D. Anderson Cancer Center: a survey of patient attitudes and opinions. J. Prosthet. Dent., 85(6):608-13, 2001.

Mehanna, H.; Paleri, V.; Robson, A.; Wight, R. \& Helliwell, T. Consensus statement by otorhinolaryngologists and pathologists on the diagnosis and management of laryngeal dysplasia. Clin. Otolaryngol., 35(3):170-6, 2010.

National Institute for Health and Clinical Excellence, (NICE). Improving outcomes in head and neck cancers. Cancer service guideline. 2004. Disponible en: www.nice.org.uk https://www.nice.org.uk/ guidance/csg6/resources/healthcare-services-for-head-and-neckcancers-pdf-2190221821

New DAHNO System. National Head and Neck Cancer Audit. 2014. Disponible en: https://files.digital.nhs.uk/publicationimport/ pub18xxx/pub18081/clin-audi-supp-prog-head-neck-dahn-1314.pdf

Newton, J. T.; Fiske, J.; Foote, O.; Frances, C.; Loh, I. M. \& Radford, D. R. Preliminary study of the impact of loss of part of the face and its prosthetic restoration. J. Prosthet. Dent., 82(5):585-90, 1999.

Ortegon, S. M.; Martin, J. W. \& Lewin, J. S. A hollow delayed surgical obturator for a bilateral subtotal maxillectomy patient: a clinical report. J. Prosthet. Dent., 99(1):14-8, 2008.

Rieger, J.; Wolfaardt, J.; Seikaly, H. \& Jha, N. Speech outcomes in patients rehabilitated with maxillary obturator prostheses after maxillectomy: a prospective study. Int. J. Prosthodont., 15(2):13944, 2002.

Rogers, S. N.; Lowe, D.; McNally, D.; Brown, J. S. \& Vaughan, E. D. Health-related quality of life after maxillectomy: a comparison between prosthetic obturation and free flap. J. Oral Maxillofac. Surg., 61(2):174-81, 2003.

Roumanas, E. D.; Freymiller, E. G.; Chang, T. L.; Aghaloo, T. \& Beumer, J., 3rd. Implant-retained prostheses for facial defects: an up to 14year follow-up report on the survival rates of implants at UCLA. Int. J. Prosthodont., 15(4):325-32, 2002.

Salinas, T. J. Prosthetic rehabilitation of defects of the head and neck. Semin. Plast. Surg., 24(3):299-308. 2010.

Salman, S. O.; Fernandes, R. P. \& Rawal, S. R. Immediate Reconstruction and Dental Rehabilitation of Segmental Mandibular Defects: Description of a Novel Technique. J. Oral Maxillofac. Surg., 75(10):2270.e1-2270.e8, 2017.

Sánchez-Ruiz, I.; González Landa, G.; Pérez González, V.; Díez Rodríguez, R.; López-Cedrún, J. L.; Miró Viar, J.; García Miñaur, S.; de Celis Vara, R. \& Sánchez Fernández, L. Tratamiento integral de las fisuras labio palatinas. Organización de un equipo de tratamiento. Cir. Pediatr., 12(1):4-10. 1999.

Schoen, P. J.; Raghoebar, G. M.; van Oort, R. P.; Reintsema, H.; van der Laan, B. F.; Burlage, F. R.; Roodenburg, J. L. \& Vissink, A. Treatment outcome of bone-anchored craniofacial prostheses after tumor surgery. Cancer; 92(12):3045-50 2001.

The glossary of prosthodontic terms: ninth edition. J. Prosthet. Dent., 117(5S):e1-105, 2017.

Hakan Tuna, S.; Pekkan, G. \& Buyukgural, B. Rehabilitation of an edentulous cleft lip and palate patient with a soft palate defect using a bar-retained, implant-supported speech-aid prosthesis: a clinical report. Cleft Palate Craniofac. J., 46(1):97-102 2009.

Weller, M. D.; Nankivell, P. C.; McConkey, C.; Paleri, V. \& Mehanna, $H$. M. The risk and interval to malignancy of patients with laryngeal dysplasia; a systematic review of case series and meta-analysis. Clin. Otolaryngol., 35(5):364-72 2010.
Correspondencia para autor:

Dr. Mauricio Vivanco Barahona Universidad de Valparaíso

Subida Leopoldo Carvallo \#211

Playa Ancha - Valparaíso

CHILE

E-mail: mauricio.vivanco@uv.cl 